\title{
Dynamic Behavior of a Predator-prey Model under the Influence of Toxin and Optimal Foraging Strategy
}

\author{
Yue Ma and Ya Li* \\ School of Mathematics and System Sciences \& LMIB, Beihang University, China
}

\begin{abstract}
Prey toxicity and coexistence of multiple prey species are properties that exist wildly in predator-prey systems. Prey toxins might inhibit the food intake and maturity of predators. Optimal foraging theory predicts that predators prefer prey which can provide more energy and cost less energy to handling food. In this project, a three-dimensional model consisting of one predator and two prey species is constructed. This model incorporates explicitly the effects of both prey toxins and the optimal foraging theory. The dynamical behaviors of the model are analyzed and the results show that prey toxins and optimal foraging strategy play important roles in the stability of the system.
\end{abstract}

Keywords-component; predator-prey model, prey toxicity, optimal foraging, local stability

\section{INTRODUCTION}

With the progress of technology and continuous expansion of industrial production, problems of environment pollution became worse in recent years. For example, pesticides are widely used in farmland to defense pests. They take effect in killing pests, while threatening the native enemy of pests at the same time. This is because the chemical residues turn pests into toxic prey for their predators [1]. Moreover, some plant species can produce toxins for self-protection, which also affect herbivores [2]. The impact of toxins on predators diet has already been stressed in a number of researches as in [3], [4] and [5]. Results indicate that the toxicity effect cannot be omitted from the system.

In [3], we achieved a toxin-determined functional response model based on the effect of toxicity on herbivore browsing. In this model, a toxin-determined functional response $g(N)$ is constructed as $g(N)=\frac{e N}{1+\widetilde{h}(N) e N}$, where the handling time $\tilde{h}(N)$ is defined by

$$
\tilde{h}(N)= \begin{cases}\frac{h}{1-a N}, & a N<1 ; \\ \infty, & a N \geq 1,\end{cases}
$$

Here $N$ is the population of prey species, $e$ is the encounter rate per unit of prey, $h$ is the handling time per unit of prey in the absence of toxins, $G$ is the toxin-adjusted maximal amount of prey a predator can ingest per unit of time. Here $1 /(4 h) \leq$ $G \leq 1 / h . a=\alpha e / G$, where $\alpha=(1-\sqrt{h G})^{2}$ is a scaling parameter which meets the condition $\max g(N)=G$.

Besides prey toxins, how predators choose proper preys based on the balance between energy intake and costs has been intensively studied. Optimal foraging theory [8] is a widely used framework for explaining and predicting the foraging behavior of predators. It predicts that predators choose prey which provide maximal net rate of energy intake, while minimizing predators' energy costs at the same time.

Kr ivan [6] studied a one predator-two prey model including optimal foraging strategy. The model assumes prey 1 is the more profitable one, i.e. $B_{1} / h_{1}>B_{2} / h_{2}$. The probability of attack prey species $i$ is $p_{i}$. It follows from the optimal foraging theory that the criteria to prey choice is to maximize the following fitness function:

$$
R\left(p_{1}, p_{2}\right)=\frac{B_{1} p_{1} e_{1} N_{1}+B_{2} p_{2} e_{2} N_{2}}{1+p_{1} h_{1} e_{1} N_{1}+p_{2} h_{2} e_{2} N_{2}} .
$$

$B_{i}$ is the conversion coefficient of consumed prey species $i$ to the biomass of predator. Let $N_{1}^{*}=\frac{B_{2}}{e_{1}\left(B_{1} h_{2}-B_{2} h_{1}\right)}$, then the monotonicity of $R\left(p_{1}, p_{2}\right)$ implies that the optimal strategy is $p_{1}=1, p_{2}=0$ for $N_{1}>N_{1}^{*}$ and $p_{2}=1$ for $N_{1}<N_{1}^{*}$. Thus, $p_{2}$ is a step function of $N_{1}$. This is a renowned result from the optimal foraging theory which describes how an generalist predator behaves when searching for food based on energymaximization rule [9], i.e. the more profitable prey is always chosen in the diet while the range of possibility of the less profitable one to be chosen relying on the population density of the more profitable prey.

Due to a number of biologically realistic uncertainties associated with predator assessment of prey encounter rates, prey profitability or predator motivational state [8], [9], there is usually considerable behavioral variation around the expected step functions. Fraxell [10] replaces the probability $p_{2}$ of attack against prey species 2 by a continuous sigmoid function of $N_{1}$, that is,

$$
\beta\left(N_{1}\right)=\frac{N_{1}^{* Z}}{N_{1}^{* Z}+N_{1}^{Z}}, Z>1
$$

$z$ is a parameter dictating the closeness of the predator diet choice to the optimal foraging. When $z$ goes to infinity, the $\beta$ is the same as the optimal foraging $p_{2}$. In addition, its continuous prosperity of this $\beta$ simplifies the analysis of the model.

All those models consider either optimal foraging strategy or prey toxins, respectively. However, the interactive impacts of diet selection and toxic prey on an ecosystem are still not clear and have not attracted enough attention. Therefore, we focus on the dynamics of a new predator-prey model which include both of those two factors in this paper. 
The paper is organized as the follows. Section 2 introduced the model with the new toxin-determined functional response, and parameter $\beta$ is used to denote the optimal foraging strategy. Sections 3 and 4 are for the stabilities ties and bifurcations of the equilibria. Section 5 is the generalization on the conclusion of the dynamical analysis of the model.

\section{THE MODEL}

Let $P=P(t)$ denotes the density of predator at time $t$, $N_{1}(t), N_{2}(t)$ denote the densities of prey species 1 and 2 . Assume that prey 1 contains toxins and prey 2 is toxin-free. We do not consider the intra-competition between two prey species.

The system in [3] is modified to include optimal strategy of predators as follows:

$$
\begin{aligned}
\frac{d N_{1}}{d t} & =r_{1} N_{1}\left(1-\frac{N_{1}}{K_{1}}\right)-P g_{1}\left(N_{1}, N_{2}\right) \\
\frac{d N_{2}}{d t} & =r_{2} N_{2}\left(1-\frac{N_{2}}{K_{2}}\right)-\beta P g_{2}\left(N_{1}, N_{2}\right) \\
\frac{d P}{d t} & =P\left(B_{1} g_{1}\left(N_{1}, N_{2}\right)+\beta B_{2} g_{2}\left(N_{1}, N_{2}\right)-D\right)
\end{aligned}
$$

where $\beta=\beta\left(N_{1}\right)$ is defined in (1).

In the above equations, $r_{i}$ and $K_{i}$ denote the intrinsic per capita growth rate and the carrying capacity of prey species $i$, respectively; $D$ is the per capita death rate of predators; $\beta\left(N_{1}\right)$ represents the probability of attack against prey 2 , which is a continuous sigmoid function of $N_{1}$. We assume $a N_{1}<1$, the functional response $g_{i}\left(N_{1}, N_{2}\right)$ is defined as

$$
g_{i}\left(N_{1}, N_{2}\right)=\frac{e_{i} N_{i}}{1+\tilde{h}_{1} e_{1} N_{1}+\beta\left(N_{1}\right) h_{2} e_{2} N_{2}},
$$

where $\tilde{h}_{1}\left(N_{1}\right)=\frac{h_{1}}{1-a N_{1}}$, and $a=\left(1-\sqrt{h_{1} G_{1}}\right)^{2} e_{1} / G_{1}$. The parameters $h_{i}, e_{i}, G_{i}$ of prey species $i$ are similar in definition with $h, e, G$ in the previous section. In the remaining of the paper, we assume that $a K_{1}<1$, so that $g_{i}\left(N_{1}, N_{2}\right)$ is always nonnegative for $N_{1} \leq K_{1}$.

\section{BOUNDARY EQUILIBRIA}

In this section, the conditions for the existence and stability of boundary equilibria of the system (2) are studied.

\section{A. Existence of Boundary Equilibria}

The equilibria of system (2) can be obtained by solving the following equations:

$$
\begin{aligned}
r_{1} N_{1}\left(1-\frac{N_{1}}{K_{1}}\right)-P g_{1}\left(N_{1}, N_{2}\right) & =0 \\
r_{2} N_{2}\left(1-\frac{N_{2}}{K_{2}}\right)-\beta\left(N_{1}\right) P g_{2}\left(N_{1}, N_{2}\right) & =0 \\
P\left(B_{1} g_{1}\left(N_{1}, N_{2}\right)+\beta\left(N_{1}\right) B_{2} g_{2}\left(N_{1}, N_{2}\right)-D\right) & =0 .
\end{aligned}
$$

There are 7 possible boundary equilibria. The first type of equilibria are those at which predator is absent, i.e., $P=0$. There are four boundary equilibria of this type:

$$
E_{0}=(0,0,0), \quad E_{1}=\left(K_{1}, 0,0\right),
$$

$$
E_{2}=\left(0, K_{2}, 0\right), \bar{E}=\left(K_{1}, K_{2}, 0\right) .
$$

It is clear that $E_{0}, E_{1}, E_{2}$ and $\bar{E}$ always exist.

The second type of boundary equilibria are those at which $P>0$. There are at most three equilibria of this type:

$$
\widetilde{E}^{ \pm}=\left(\widetilde{N}_{1}^{ \pm}, 0, \widetilde{P}^{ \pm}\right), \widehat{E}=\left(0, \widehat{N_{2}}, \widehat{P}\right),
$$

with

$$
\begin{aligned}
\widetilde{N}_{1}^{ \pm} & =\frac{B_{1} e_{1}+D\left(a-h_{1} e_{1}\right) \pm \sqrt{\Delta}}{2 B_{1} e_{1} a}, \\
\tilde{P}^{ \pm} & =r_{1} \widetilde{N}_{1}\left(1-\frac{\widetilde{N}_{1}^{ \pm}}{K_{1}}\right) \frac{B_{1}}{D}, \\
\widehat{N}_{2} & =\frac{D}{\left(B_{2}-h_{2} D\right) e_{2}}, \\
\hat{P} & =\frac{r_{2} B_{2}\left[e_{2} K_{2}\left(B_{2}-h_{2} D\right)-D\right]}{K_{2} e_{2}{ }^{2}\left(B_{2}-h_{2} D\right)^{2}} .
\end{aligned}
$$

Where $\Delta=\left[B_{1} e_{1}+D\left(a-h_{1} e_{1}\right)\right]^{2}-4 B_{1} e_{1} a D$.

Let

$$
\begin{aligned}
w_{i} & =B_{i} G_{i}-D, i=1,2, \\
w_{K 1} & =B_{1} g_{1}\left(K_{1}, 0\right)-D \\
w_{K 2} & =B_{2} g_{2}\left(0, K_{2}\right)-D \\
\bar{w} & =B_{1} g_{1}\left(K_{1}, K_{2}\right)+B_{2} g_{2}\left(K_{1}, K_{2}\right)-D .
\end{aligned}
$$

All of the above quantities are biologically meaningful. More specifically, $w_{i}$ represents the maximum fitness of predator when only prey species $i$ is present, $w_{K 1}$ represents the predator's fitness when $\left(N_{1}, N_{2}\right)=\left(K_{1}, 0\right), w_{K 2}$ is the predator's fitness when $\left(N_{1}, N_{2}\right)=\left(0, K_{2}\right)$, and $\bar{w}$ represents the predator's fitness when $\left(N_{1}, N_{2}\right)=\left(K_{1}, K_{2}\right)$.

Note that the value of $g_{1}\left(N_{1}, 0\right)$ is increasing as $N_{1}$ is increasing from zero, until it achieves the maximum value $G_{1}$ at $N_{1 m}=\frac{1}{a+\sqrt{h_{1} e_{1} a}}$, and then decreases for $N_{1}$ starting from $N_{1 m}$. The function $g_{2}\left(0, N_{2}\right)$ is a monotonically increasing function of $N_{2}$. In the remaining of the paper, we assume that $N_{1 m}<K_{1}$ and $g_{2}\left(0, K_{2}\right)<G_{2}$. Under those assumptions, we have: $w_{K i}<$ $w_{i}$.

The existence results below suggest that for the predator to be able to survive in the system, its maximum fitness must be positive.

Theorem 1. Let $w_{i}$ and $w_{K i}$ be as defined in (6).

a) $\tilde{E}^{ \pm}$does not exist if $w_{1}<0$; if $w_{K 1}>0$, then $\tilde{E}^{-}$exists but $\widetilde{E}^{+}$does not; both $\widetilde{E}^{+}$and $\tilde{E}^{-}$exist when $w_{K 1} \leq 0 \leq w_{1}$, with $\widetilde{E}^{+}=\widetilde{E}^{-}$if $w_{1}=0$.

b) $\hat{E}$ exists if and only if $w_{2}>0$.

Proof. The proof for $\widetilde{E}^{ \pm}$is identical with the proof in Theorem 3.1 of [1]. Figure 1 shows graphically the existence of those equilibria. We only provide the proof for the existence of $\widehat{E}$. 

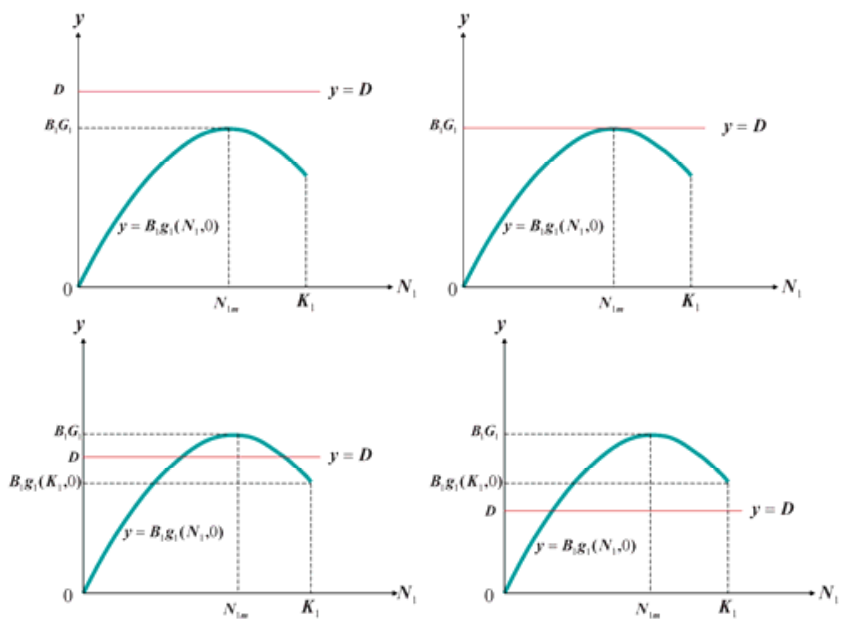

FIGURE I. PLOT OF $y=B_{1} g_{1}\left(N_{1}, 0\right)$ AND $y=D$.EVERY INTERSACTION OF TWO CURVES PRESENTS AN EQUILIBRIUM.

Note that $\beta\left(N_{1}\right)=1$ if $N_{1}=0$, so $\hat{E}$ can be calculated by solving the equations:

$$
B_{2} g_{2}\left(0, N_{2}\right)=D, r_{2} N_{2}\left(1-\frac{N_{2}}{K_{2}}\right)=P g_{2}\left(0, N_{2}\right)
$$

From the second equation of (7), we have $P=\frac{r_{2} N_{2}\left(1-\frac{N_{2}}{K_{2}}\right)}{g_{2}\left(0, N_{2}\right)}$.

Therefore, $P>0$ if and only if $0<N_{2}<K_{2}$. It follows that we only need to show that the first equation of (7) has a solution between 0 and $K_{2}$. Since $g_{2}\left(0, N_{2}\right)$ is monotonically increasing with respect to $N_{2}$, and $B_{2} g_{2}(0,0)=0<D$, $B_{2} g_{2}\left(0, K_{2}\right)=w_{2}+D>D$, it follows that there must exist a solution $\widehat{N_{2}} \in\left(0, K_{2}\right)$. This completes the proof.

\section{A. Stability of Boundary Equilibria}

The stability conditions of the four equilibria listed in (4) can be easily obtained by calculating the eigenvalues of the Jacobian matrices at the equilibria. The results are summarized in the following theorem.

Theorem 2. Let $\bar{w}$ be as defined in (6). Then

a) $E_{0}, E_{1}$ and $E_{2}$ are always unstable;

b) $\bar{E}$ is locally asymptotically stable if $\bar{w}<0$.

The stability results of the equilibria $\widetilde{E}^{ \pm}$are given below.

Theorem 3. Let

$$
w_{1}>0, \text { and } G_{1}<\frac{1}{4 h_{1}} \text {. }
$$

a) The equilibrium $\tilde{E}^{+}$is always unstable;

b) There exist constants $\sigma_{1}>0$ and $w_{1 c}>0$, such that $\tilde{E}^{-}$ is l.a.s. if $\frac{r_{1}}{r_{2}}>\sigma_{1}$ and $w_{1}>w_{1 c}$, and unstable if either $\frac{r_{1}}{r_{2}}<\sigma_{1}$ or $w_{1}<w_{1 c}$; c) When $\frac{r_{1}}{r_{2}}>\sigma_{1}$, a Hopf bifurcation occurs at $w_{1}=w_{1 c}$, and stabile periodic solutions exist for $w_{1}$ near $w_{1 c}$ and $w_{1}<$ $w_{1 c}$.

Proof. The Jacobian matrix at the equilibrium point $\widetilde{E}^{ \pm}$is

$$
\tilde{J}^{ \pm}=\left(\begin{array}{ccc}
a_{1}^{ \pm} & * & a_{2} \\
0 & a_{3}^{ \pm} & 0 \\
a_{4}^{ \pm} & * & 0
\end{array}\right)
$$

where

$$
\begin{aligned}
& a_{1}^{ \pm}=r_{1}\left(1-\frac{2 \widetilde{N}_{1}^{ \pm}}{K_{1}}\right)-\widetilde{P}^{ \pm} \frac{\partial g_{1}}{\partial N_{1}}\left(\widetilde{N}_{1}^{ \pm}, 0\right), \\
& a_{2}=-\frac{D}{B_{1}} \\
& a_{3}^{ \pm}=r_{2}-\widetilde{P}^{ \pm} \frac{D e_{2} \beta\left(\widetilde{N}_{1}^{ \pm}\right)}{B_{1} e_{1} \widetilde{N}_{1}^{ \pm}}, \\
& a_{4}^{ \pm}=\tilde{P}^{ \pm} \frac{\partial g_{1}}{\partial N_{1}}\left(\widetilde{N}_{1}^{ \pm}, 0\right),
\end{aligned}
$$

and the other two entries have no effect on the stability of the equilibrium, which are denoted.

The matrix $\tilde{J}^{ \pm}$has one eigenvalue $a_{3}^{ \pm}$and two other eigenvalues are the same as that of the matrix

$$
\widetilde{M}^{ \pm}=\left(\begin{array}{cc}
a_{1}^{ \pm} & a_{2} \\
a_{4}^{ \pm} & 0
\end{array}\right)
$$

Thus, $\tilde{E}^{ \pm}$is 1.a.s. if and only if $a_{2} a_{4}^{ \pm}<0, a_{3}^{ \pm}<0, a_{1}^{ \pm}<0$.

Since $\tilde{P}^{+}>0$ and $\frac{\partial g_{1}}{\partial N_{1}}\left(\widetilde{N}_{1}^{+}, 0\right)<0$, then $a_{2} a_{4}^{+}>0$. So $\widetilde{E}^{+}$ is always unstable, and part (a) is proved.

Next, we consider the stability of $\tilde{E}^{-}$.

Since $\widetilde{P}^{-}>0$ and $\frac{\partial g_{1}}{\partial N_{1}}\left(\widetilde{N}_{1}^{-}, 0\right)>0$, we have $a_{2} a_{4}^{-}<0$.

Substitution of $\tilde{P}^{-}=r_{1} \widetilde{N}_{1}^{-}\left(1-\frac{\widetilde{N}_{1}^{-}}{K_{1}}\right) \frac{B_{1}}{D}$ in $a_{3}^{-}$yields $a_{3}^{-}=$ $r_{2}-\frac{r_{1} e_{2} \beta\left(\widetilde{N}_{1}^{-}\right)}{e_{1}}\left(1-\frac{\widetilde{N}_{1}^{-}}{K_{1}}\right)$. Let $\sigma_{1}=\frac{e_{1}}{e_{2} \beta\left(\widetilde{N}_{1}^{-}\right)\left(1-\frac{\widetilde{N}_{1}^{-}}{K_{1}}\right)}>0$, where $\widetilde{N}_{1}^{-}$ is given in (5) and is independent of $r_{1}$ and $r_{2}$. Then $a_{3}^{-}<0 \Leftrightarrow$ $\frac{r_{1}}{r_{2}}>\sigma_{1}$.

The discussion about the sign of $a_{1}^{-}$is the same as that of the entry $b_{3}^{-}$in Theorem 3.3 of [1], except that the parameter $a_{1}$ in that theorem is replaced by $a, \sigma_{1 c}$ is replaced by $\sigma_{1}$ in this paper. Therefore, the remaining proof is the same and we omit it in this paper.

The stability of $\hat{E}$ is stated in the following Theorem, which can be proved in a similar way as for $\tilde{E}^{ \pm}$.

Theorem 4. Let $w_{2}>0, G_{2}<\frac{1}{4 h_{2}}$. 
a) There exist constants $\sigma_{2}>0$ and $w_{2 c}>0$, such that $\hat{E}$ is l.a.s. if $\frac{r_{2}}{r_{1}}>\sigma_{2}$ and $w_{2}>w_{2 c}$, and unstable if either $\frac{r_{2}}{r_{1}}<\sigma_{2}$ or $w_{2}<w_{2 c}$;

b) When $\frac{r_{2}}{r_{1}}>\sigma_{2}$, a supercritical Hopf bifurcation occurs at $w_{2}=w_{2 c}$.

We remark that the sets of stability conditions (see, for example, (8)) identified in this section are sufficient but not necessary. There are other scenarios in which the equilibria $\widetilde{E}^{-}$ and $\hat{E}^{-}$may be stable. Nonetheless, these results provide useful insights into the role of prey toxicity and optimal foraging strategy in the composition of prey community. For example, Theorem 3 suggests that in the presence of predator, if prey 1 has a relatively high growth rate $\left(r_{1} / r_{2}>\sigma_{1}\right)$ and higher toxicity (smaller $G_{1}$ value, see (8)), then it is possible for prey 1 to exclude prey 2 (as $\tilde{E}^{-}$is 1.a.s.). Consider the system of [1], we can observe that, if we do not consider the competition effect between prey species, i.e., let $\beta_{12}=0$, then $\sigma_{1 c}=$ $\frac{e_{1}}{e_{2}\left(1-\frac{\tilde{N}_{1}^{-}}{K_{1}}\right)}$. The equilibrium $\tilde{E}^{-}$is l.a.s. if $\frac{r_{1}}{r_{2}}>\sigma_{1 c}$. If we include the optimal foraging parameter $0<\beta\left(N_{1}\right)<1$ in the system, then $\sigma_{1 c}$ is replaced by $\sigma_{1}$. Since $\sigma_{1}>\sigma_{1 c}$, Theorem 3 implies that optimal foraging strategy may prevent the extinction of toxin-free plant species.

\section{INTERIOR EQUILIBRIUM}

In this section, we derive the conditions in which an interior equilibrium exists. Most of the conditions are motivated by biological consideration.

\section{A. Existence of an Interior Equilibrium}

An interior equilibrium of the system (2) is indicated by $E^{*}=\left(P^{*}, N_{1}^{*}, N_{2}^{*}\right)$, i.e., all components of $E^{*}$ are positive due to their biological purpose. Then $N_{1}^{*}$ and $N_{2}^{*}$ satisfy the equations:

$$
\begin{aligned}
& B_{1} g_{1}\left(N_{1}, N_{2}\right)+\beta\left(N_{1}\right) B_{2} g_{2}\left(N_{1}, N_{2}\right)-D=0, \\
& \frac{r_{1}}{e_{1}}\left(1-\frac{N_{1}}{K_{1}}\right)-\frac{r_{2}}{\beta\left(N_{1}\right) e_{2}}\left(1-\frac{N_{2}}{K_{2}}\right)=0 .
\end{aligned}
$$

Let

$$
N_{2}^{\circ}=K_{2}\left(1-\frac{r_{1} e_{2}}{r_{2} e_{1}}\right), w_{2}^{\diamond}=B_{2} g_{2}\left(0, N_{2}^{\circ}\right)-D .
$$

Theorem 5. Assume $\frac{r_{1}}{r_{2}}<\frac{e_{1}}{e_{2}}$ holds. System (2) has an interior equilibrium $E^{*}=\left(P^{*}, N_{1}^{*}, N_{2}^{*}\right)$, if $\bar{w} w_{2}^{\circ}<0$.

Proof. Note that all components of $E^{*}$ are positive, if exists, and they must satisfy the equations of (9).

We can solve for $N_{2}$ as a linear function of $N_{1}$ :

$$
N_{2}=K_{2}\left[1-\frac{r_{1} e_{2} \beta\left(N_{1}\right)}{r_{2} e_{1}}\left(1-\frac{N_{1}}{K_{1}}\right)\right]:=\phi\left(N_{1}\right) \text {, }
$$

By replacing $\phi\left(N_{1}\right)$ for $N_{2}$, the first equation in (9) can be re-written as: $Q\left(N_{1}\right):=B_{1} g_{1}\left(N_{1}, \phi\left(N_{1}\right)\right)+B_{2} g_{2}\left(K_{1}, K_{2}\right)-D$.
From (10) and (11), we have $Q(0)=w_{2}^{\circ}$ and $Q\left(K_{1}\right)=\bar{w}$. Clearly, $\bar{w} w_{2}^{\circ}<0$ suggests that there exists an $N_{1}^{*} \in\left(0, K_{1}\right)$, such that $Q\left(N_{1}^{*}\right)=0$.

With $N_{2}^{*}=\phi\left(N_{1}^{*}\right)$, then $0<K_{2}\left[1-\frac{r_{1} e_{2}}{r_{2} e_{1}}\right]<N_{2}^{*}<K_{2}$. And $P^{*}=r_{1}\left(1-\frac{N_{1}^{*}}{K_{1}}\right) \frac{N_{1}^{*}}{g_{1}\left(N_{1}^{*}, N_{2}^{*}\right)}>0$.

Therefore, all the factors in $E^{*}$ are positive. $E^{*}$ is an interior equilibrium of the system (2).

Notice that only prey toxicity level has some affect the existence of interior equilibrium, since $\bar{w}, w_{2}^{\diamond}$ are both related to toxins. The parameter $\beta\left(N_{1}\right)$ can be found in (11), which indicates that optimal foraging strategy play an important role in determining the population density of prey species 2 .

\section{B. Stability of $E^{*}$}

According to Routh-Hurwitz Theorem, the local stability of $E^{*}$ depends on the coefficients of characteristic equation of its Jacobian matrix. Suppose the characteristic equation:

$$
a_{3} s^{3}+a_{2} s^{2}+a_{1} s+a_{0}=0
$$

If all coefficients are positive, and $a_{1} a_{2}-a_{0} a_{3}>0$. then the interior equilibrium is locally asymptotically stable.

Due to the express of the new functional response of our model, all those coefficients have very complicated forms. Hence, the stability conditions cannot be expressed in a simple way. However, numerical simulations (see Figure 2) show that there might exist a stable interior equilibrium of the system under suitable parameter values.
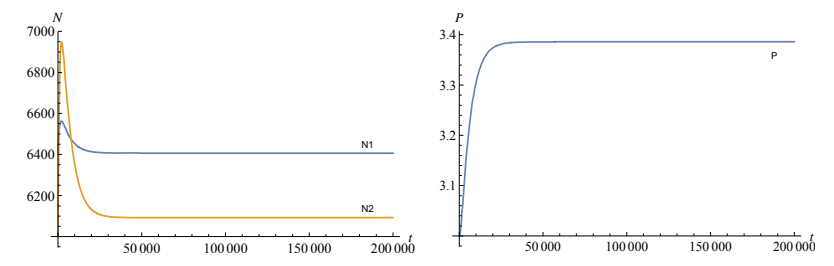

FIGURE II. DENSITIES OF TWO PREY SPECIES AND \& DENSITY OF PREDATOR SPECIE(THE PARAMETERS ARE

$r_{1}=0.00167 ; r_{2}=0.0028 ; K_{1}=8000 ; K_{2}=15000 ; e_{1}=0.0001 ; e_{2}=0.0005$; $h_{1}=1 / 10 ; h_{2}=1 / 160 ; B_{1}=0.00001 ; B_{2}=0.000036 ; G=8 ; z=50$; $D=0.000114)$

\section{DisCUSSION}

In this paper, we integrate diet selection theory for an adaptive predator with a toxin-determined functional response $g_{i}\left(N_{1}, N_{2}\right)$ in a one predator-two prey model. Results show that both of those factors play important roles in the dynamics of the system. The critical existence threshold such as $w_{i}, w_{2}^{\circ}$ implies that toxicity level may play a key role in the existence of equilibria.

Analysis also shows that optimal foraging strategy may help preventing the toxin-free plant species from extinction.

\section{REFERENCES}

[1] Qihua Huang, Hao Wang, Mark A. Lewis, The impact of environmental toxins on predator-prey dynamics. Journal of Theo. Bio., Vol. 378, Aug. 2015, 12-30. 
[2] R.T.Palo, C.T.Robbins, Plant chemical defenses against mammalian herbivory, CRC Press, Boca Raton, Florida, 1991.

[3] Y. Li, Z. Feng, "Dynamics of a plant-herbivor model with toxin-induced functional response", Math. Biosci. Eng., Vol. 7, Jan. 2010, 151-174.

[4] Y. Li, Z. Feng, R. Swihart, J. Bryant and N. Huntly, "Modeling the impact of plant toxicity on plant-herbivore dynamics", J. Dyn. Diff. Eq., vol. 18, 2006, 1021-1042.

[5] Tansky, M., "Switching effect in predator-prey system", J. Theor. Biol., vol. 70, 1978, 263-271.

[6] Vlastimal Krivan, "Optimal foraging and predator-prey dynamics", Theoretical Population Biology, vol. 49, 1996, 265-290.

[7] Colombo, R., and Krivan, V., "Selective strategies in food webs", IMA J of Math. Appl. Medicine Biol., vol. 10, 1993, 281-291.

[8] Stevens, D.W., How important are partial preferences? Anim. Behav., Vol. 33, 1985, 667-9.

[9] M. Westoby, "What are the biological bases of varied diets?", Am. Nat., vol. 112, 1978, 627-631.

[10] J. M. Fryxell, P. Lundberg, "Diet choice and predator-prey dynamics , Evolutionary Ecology”,Evolutionary Ecology, vol. 8, 1994, 407-421.

[11] Krebs,J.R. and MacCleery, R.H. Optimization in behavioural ecology. Blackwell, Oxford. Behavioral ecology, 1984.

[12] Stephens, D.W. and Krebs, J.R., Foraging Theory, Princeton University Press, 1986. 